\title{
Global Patterns and Country Experiences with the Formulation and Implementation of Food-Based Dietary Guidelines
}

\author{
Janice Albert \\ Nutrition and Consumer Protection Division, Food and Agriculture Organization, Rome, Italy
}

\section{Key Words}

Food-based dietary guidelines, global · Nutrition education • National dietary guidelines - Dietary guidelines •

$\mathrm{FAO}$, developing country diets

\begin{abstract}
Nutrition information and advice for the public must be credible from a scientific perspective and easy to understand and follow. The Food and Agriculture Organization of the United Nations (FAO) has promoted the preparation and use of food-based dietary guidelines (FBDG) in Asia, the Near East, Latin America and the Caribbean and Africa. The paper is based on a survey of 28 developing countries and case studies of South Africa, India, Chile and Guatemala, which were conducted by FAO in 2004. All FBDG stress the importance of dietary variety and balance, promote fruit and vegetable consumption, discourage excessive salt, sugar and alcohol consumption, and promote physical activity. In other respects, FBDG vary according to the specific health, behavior, culture and economic conditions within a country. The steps used in developing and implementing FBDG are discussed, with country examples to illustrate achievements, mistakes and obstacles. Main areas for improvements are: (1) FBDG are mainly developed and used within the health sec-
\end{abstract}

The views expressed in this publication are those of the author and do not necessarily reflect the views of the Food and Agriculture Organization of the United Nations.

\section{KARGER}

Fax +4161306 1234

E-Mail karger@karger.ch

www.karger.com
(C) $2007 \mathrm{~S}$. Karger AG, Basel

$0250-6807 / 07 / 0518-0002 \$ 23.50 / 0$

Accessible online at:

www.karger.com/anm tor, involvement of the agriculture and education sectors is needed; (2) few counties communicate to widely reach the public; (3) many messages do not motivate changes in behavior, and (4) the application of FBDG in setting policies not sufficient received attention. Copyright $\odot 2007$ S. Karger AG, Basel

\section{Background/Aims}

There is keen public interest in nutrition in many countries and it is important that the information and advice that the public receives is credible from a scientific perspective and easy to understand and follow. Foodbased dietary guidelines (FBDG) aim to provide this type of information to the general public. FBDG use common language and are intended for use by the average person; they should not be confused with recommended nutrient intakes which provide quantitative recommendations for use by specialists for ensuring the health of populations [1]. While recommended nutrient intakes are standards which apply worldwide, FBDG are specifically designed for each country and advice can differ according to the needs of the particular population. FBDG are also a policy tool, which can be used to coordinate nutrition programs and to evaluate the adequacy of the food supply in meeting the nutritional needs of the population [2].

The Food and Agriculture Organization of the United Nations (FAO) and the World Health Organization 
(WHO) have promoted the notion that governments should provide nutritional advice to the public which is relevant for different age groups and lifestyles, and culturally appropriate to the country's population [3]. In 1995, FAO and WHO sponsored an Expert Consultation on the Preparation and Use of Food-Based Dietary Guidelines [4]. The technical report of this meeting provided the rationale for FBDG and an overview of the steps involved in producing dietary guidelines. After the meeting, FAO sponsored 11 workshops in which more than 95 countries participated. WHO promoted the concept of FBDG, primarily through its regional offices. The two UN agencies have trained nutritionists and facilitated the development of FBDG in Asia, the Near East, Latin America and the Caribbean and Africa.

This aim of this paper is to describe current global patterns in the contents of FBDG and to analyze countries' experiences with the formulation and implementation of FBDG.

\section{Method}

Recognizing the large gaps in available information about the development and uses of FBDG in the developing world, FAO initiated a pilot study of FBDG in August 2004. Our research included a survey and four of case studies.

Survey

A questionnaire containing broad, open-ended questions was prepared in English and Spanish and sent by e-mail to 42 countries. These countries were chosen because they had sent government staff to participate in FAO workshops on FBDG. Twentyeight countries responded to the survey (response rate of $67 \%$ ).

Government representatives were asked if they had developed FBDG and food guides, what messages were contained in the FBDG, which organizations and individuals were involved in preparation of the FBDG, how the FBDG were being used in the country, if there were problems in using the FBDG, and whether the FBDG had been evaluated or there were plans to evaluate their use.

\section{Case Studies}

Five experts in nutrition education from India, South Africa, Chile and Guatemala were commissioned to provide case studies of their countries' experiences in producing and disseminating FBDG. These countries were chosen because they have highly diverse populations and a range of very challenging nutrition education needs. The experts used a range of methods including faceto-face interviews, telephone interviews, surveys and review of documentation. Since four of the experts had been directly involved in the development of their countries' FBDG, they were also able to draw upon personal in-depth knowledge of the processes followed.

FBDG Global Patterns and Country Experiences

\section{Results}

The information provided below is based on the research study about FBDG, as well as FAO experiences in assisting countries in developing their FBDG and food guides.

\section{Global Patterns}

Throughout the world, there are several characteristics commonly found in national dietary guidelines. First, the importance of variety and balance are always stressed in FBDG. Second, it is very common for FBDG to promote increased fruit and vegetable consumption. Third, FBDG often include advice to discourage excessive salt, sugar and alcohol consumption. Fourth, with increased concern about sedentary lifestyles, physical activity messages are becoming more common.

Close examination of dietary guidelines demonstrates that there are significant differences among FBDG. This is not surprising since FBDG are determined by the specific health, behavior, culture and economic conditions within a country. For example, advice about dietary fats and carbohydrates may vary greatly, according to the nutritional problems in a country.

Providing one set of guidelines for an entire nation is challenging and sometimes controversial. Recognizing the different nutritional needs during the life cycle, a few countries have created separate dietary guidelines for different age groups (e.g. Brazil); while others have incorporated advice for specific target groups in their general guidelines (e.g. Bangladesh and Nigeria). In very large countries with extremely diverse populations, the notion of separate guidelines for different segments of the population has arisen at times. Interestingly, all of the countries in our study, no matter how large and diverse, had created FBDG which were intended for everyone. Whether these general FBDG have been effective among different population groups is a matter for future research. The presentation of the messages varies as well, some countries have very short simple messages while others give fairly detailed advice. To view the diversity of FBDG and food guides from around the world, see the FAO website: http://www.fao.org/ag/ agn/nutrition/education_guidelines_en.stm.

\section{Process of Developing FBDG}

FAO and WHO recommend a series of steps to develop FBDG. Each step is important for the efficient and effective development of dietary guidelines (fig. 1). The 
Fig. 1. Recommended steps for developing and implementing FBDG.

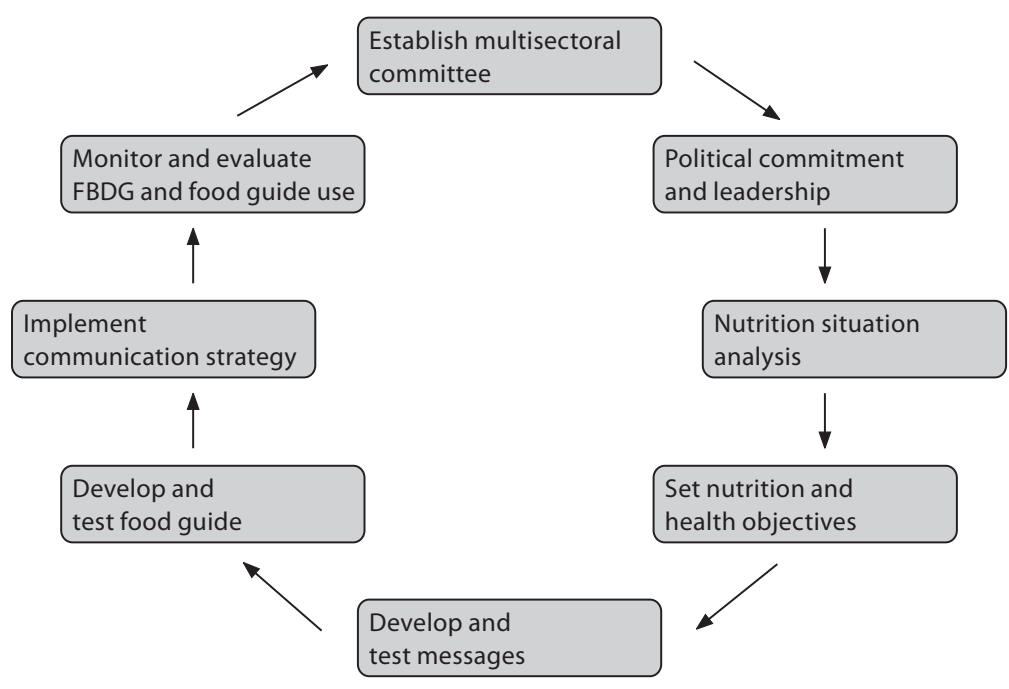

process is dynamic: dietary guidelines and food guides should not considered to be permanent. They are tools which should be reassessed periodically to ensure that they are always up to date and relevant to the public's needs. The process applies methods that can seem overwhelming and complicated. However, experiences from many types of countries show that the challenges of developing FBDG can be met even in countries which lack extensive resources.

\section{Multisectoral Committee}

A first step in developing FBDG is the formation of a national committee which includes representatives of the leading ministries (e.g. health, agriculture, education, social welfare) and others who can assist in developing and promoting FBDG such as scientific societies, food industry associations and consumer organizations. While including a range of institutions is essential, experience shows that FBDG development is most successful when there is a leading individual who is able to dedicate time to advocating the concept of FBDG and coordinating the process.

Example - Guatemala: When Guatemala began work on its dietary guidelines in 1995, they formed a commission with support from the Department of Nutrition within the Ministry of Public Health and Social Assistance. Schools of education, a pediatric hospital, the De- partment of Maternal and Child Health, the Ministry of Education, the Ministry of Agriculture, Ranching and Food as well as the PAHO Institute of Nutrition for Central America and Panama were members of the commission. The multisectoral team met over a 2 -year period to review evidence about the nutrition situation and to decide on the main objectives of the guidelines. They approved the messages and food guide and committed their institutions to promoting the dietary guidelines [5].

\section{Political Support and Commitment}

Without political support and coordination, bureaucracies may not incorporate the FBDG into their programs or devote resources to developing and promoting the FBDG. In a number of countries, the Parliament approves the FBDG and the Prime Minister or President announces the guidelines.

Example - South Africa: South Africa's experience illustrates the importance of involving all the relevant government agencies and stakeholders at the earliest stages in developing FBDG. In 1997, the Nutrition Society of South Africa began developing FBDG. A committee included universities, the food industry, health profession associations, a UN agency and the Directorate of Nutrition within the Ministry of Health. Participation in the committee was voluntary and participation from some representatives was low [6]. Before the FBDG were ap- 
proved by the Parliament, a public controversy arose over some aspects of the recommendations regarding meat and carbohydrates. This delayed the approval of the FBDG until 2003. Better participation and involvement of all the relevant government units and stakeholders earlier in the process might have prevented this delay.

\section{Analysis of Nutrition Situation and Setting of Objectives}

The next steps are technical: the analysis of the nutrition situation and identification of objectives is carried out by nutritionists and other specialists. Using data such as health and census statistics, household expenditure and food consumption surveys, food balance sheets, and the scientific literature, the technical analysis leads to identification of the nutrition issues which are significant for public health. Most countries do not have good, comprehensive data for formulating their FBDG and the lack of data can cause long delays in developing FBDG.

Example - Namibia: Namibia's experiences with developing their FBDG illustrate how a country coped with the lack of information. In 1998, FAO provided technical assistance to Namibia for the formulation of their guidelines. The Health Ministry did not have information about food consumption. To carry out their analysis, they relied on data on household expenditures and the observations of medical personnel to obtain information about the most important health problems and priorities [7].

The positive lesson from Namibia is that the country found a way to cope with the limitations in their resources and followed the recommended steps to the extent possible. They did not allow an imperfect situation to stop their FBDG development.

\section{Testing of Messages and Food Guides}

The general public has difficulty understanding the words and concepts which are used by nutritionists. Even after the guidelines are developed, the words used in public information campaigns may have to be modified to be understood by different audiences [8].

Some concepts which are commonly used by nutritionists are not easily conveyed to all the segments of the public. For example, dietary variety and portion size are basic concepts in nutrition but they are often misunderstood. Consumers may not understand the amounts conveyed by words such as 'sparingly', 'increase', 'moderate', and visual symbols and common measurements can assist them. The testing of messages and food guides is a crucial step in the formulation of FBDG. Unfortunately, countries may omit this step due to lack of awareness of its importance or lack of resources to carry out this type of research. Regardless of the amount of expertise and experience existing within a team, pre-testing the messages and food guides always brings valuable new information.

Example - Thailand: Before Thailand published its FBDG and Nutrition Flag in 1998, they tested the food guide and messages. The public was asked about the $\mathrm{Nu}$ trition Flag to assess their understanding of portion size and quantities. Thais developed the 'rice-serving spoon' as the household unit for measuring foods. Using this flat broad spoon, the research team obtained realistic estimates of portions eaten and a way to explain quantities to the public. Hundreds of people were interviewed in department stores, food markets, factories and universities. Since travel to rural areas was difficult, the researchers overcame this problem by interviewing commuters from rural areas at bus stations [9].

\section{Communication Strategy and Dissemination of Information}

A major obstacle in the implementation of FBDG is that nearly all countries lack sufficient resources to develop and disseminate FBDG-related information. Without a communication strategy, the FBDG materials may not reach a wide audience and they may have a very limited impact. Education and communication expertise, as well as nutrition expertise, is needed for the successful formulation and implementation of FBDG. FBDG must be widely distributed and community workers must be trained to use the materials.

Example - India: When India published its dietary guidelines in 1998, the materials were translated into 6 Indian languages to reach the diverse population and there was newspaper, radio and television coverage in the various regions of the country. India used its extensive health, agriculture and education networks for disseminating information. Professionals and community workers received training about the FBDG. Non-governmental organizations and professional societies also promoted the guidelines. Special events such as 'national nutrition week' and 'agriculture field days' were used to promote the guidelines [10]. A key lesson is that countries should use the well-established channels of communication and existing training activities to promote the FBDG. 


\section{Monitoring and Evaluation}

The process of disseminating FBDG and food guides should be assessed to ensure that the messages are reaching their audiences. Without evaluation, it is difficult to demonstrate that the messages and food guide have an impact on consumer awareness and to know whether knowledge, attitudes and behaviors are changing. The evaluations should take into consideration the environment and potential for change within realistic time frames. Expertise is needed to conduct an evaluation of FBDG. Many countries in our survey had not conducted evaluations and did not know how to carry out this activity.

Example - Chile: Chile is one of the few countries which has evaluated its dietary guidelines and food guide. The evaluation included different types of institutional settings and population groups. A study in a clinic found that patients with diet-related chronic diseases received messages to eat more fruits and vegetables but had not heard about reducing animal fats. A study in schools found that children had seen the pyramid but did not understand the portions. A third study surveyed consumers over the Internet. Initially, only $30 \%$ of the consumers knew about the guidelines and $60 \%$ knew about the food pyramid. After the same consumers received information directly, the awareness and willingness to change diets rose to $80 \%$ [11]. The information obtained from the evaluation was used to revise the FBDG in 2005.

\section{Limitations in Current Knowledge}

Although many countries have formulated and implemented FBDG, our current state of knowledge about these tools is very limited and there is a need for more research on dietary guidelines. Unfortunately, much of the information about the formulation and implementation of dietary advice is unavailable to the scientific community. During our pilot study, our literature search of major data bases for nutrition, health and agricultural publications identified only 50 articles which specifically addressed the topic of dietary guidelines. Few nutritionists have published information about their country's dietary guidelines in the international scientific journals. Often the papers which exist explain the messages but do not discuss how guidelines were formulated and are being used. While governments publish their guidelines, they are difficult to find and most information is published in languages which are not widely understood internationally.

\section{Future Work}

In our review of experiences with developing and implementing FBDG in different countries, we have found a number of problems. There are four main issues which could lead to improvements in the formulation and implementation of FBDG. First, our survey found that FBDG are mainly developed and used within the health sector. There is a need for greater involvement of the agriculture and education sectors. Second, few counties have a communication strategy or make use of all the ways to reach the public. Many messages do not consider the reasons people choose foods and ways to motivate changes in behavior. Fourth, FBDG are intended to assist policymakers, as well as being educational tools. However, the application of FBDG in setting policies appears to be an area which has not received attention by researchers or practitioners.

The process of developing and implementing FBDG can seem to be a daunting exercise; however, countries have found ways to overcome many obstacles. FBDG are evolving and nutritionists can benefit from sharing experiences with other countries. To make information more accessible to the scientific community and practitioners, FAO is developing a website which will provide information about FBDG and food guides around the world. In addition, we will continue to provide scientific advice and technical assistance to countries in their efforts to provide nutrition information to the public.

\section{Acknowledgements}

The author expresses appreciation to the government participants in the survey for taking time to share their experiences with FAO. In addition, the following individuals contributed to the research: Veronika Molina, Sonia Olivares, Penny Love, Anne Behr and Lalita Bhattacharjee produced case studies; Kraisid Tontisirin, William Clay, Ellen Muehlhoff, Peter Glasauer, Cecilio Morón, Dirk Schulz and Biplab Nandi contributed expertise and contacts, and Joanna Lyons, Jayne Beaney, Jenny E. Martinez Nocito and Gretchen Dueñas assisted in the research. The International Life Sciences Institute contributed financial support for the pilot research. 


\section{References}

1 Taylor CL, Albert J, Weisell R, Nishida C: International dietary standards: FAO and WHO; in Bowman B, Russell R (eds): Present Knowledge in Nutrition, ed 9. Washington, International Life Sciences Institute, vol II, pp 876-887.

2 Schneeman BO: Preparation and use of food-based dietary guidelines: lessons from Thailand and the Philippines. Food Nutr Agric 2001;28:55-64.

3 FAO: International Conference on Nutrition, Plan of Action for Nutrition. Rome, FAO/WHO, 1992.
4 FAO/WHO: Preparation and Use of FoodBased Dietary Guidelines. Report of a Joint FAO/WHO Consultation. Geneva, WHO, 1998.

5 Molina V: Estudio de Caso: Proceso de Elaboracion e Implementacion de las Guias Alimentarias de Guatemala. Guatemala, INCAP/OPS, Oct 2004 (unpubl study).

6 Love P, Behr A: The South African Dietary Guidelines: Country Report. FAO, Oct 2004 (unpubl study).

7 Glasauer P: FAO Technical Backstopping Officer to the Joint Initiative of FAO, WHO, UNICEF and the Namibian Ministry of Agriculture, Water and Rural Development, Ministry of Basic Education and Culture, Ministry of Health and Social Services and University of Namibia 1998-2000 (pers commun).
8 Clay W: Preparation and use of food-based dietary guidelines. Food Nutr Agric 1997; 19: 42-47.

9 Dueñas G: Pretesting methods for dietary guidelines and food guide models: experiences of South Africa and Thailand. FAO, Aug 2005 (unpubl study).

10 Bhattacharjee L: Food-based dietary guidelines: case study from India. FAO, Oct 2004 (unpubl study)

11 Olivares S: Guías Alimentarias Basadas en Alimentos: La Experiencia de Chile, FAO, Oct 2004 (unpubl study). 